\title{
High-mobility group box 1 released from astrocytes promotes the proliferation of cultured neural stem/progenitor cells
}

\author{
MAN LI ${ }^{1}$, LIN SUN $^{2}$, YONG LUO $^{1,3}$, CHENCHEN XIE $^{1,3}$, YUESHAN PANG $^{1,3}$ and YUAN LI ${ }^{4}$ \\ ${ }^{1}$ Department of Neurology, The First Affiliated Hospital of Chongqing Medical University, Chongqing 40016; \\ ${ }^{2}$ Department of Orthopedics, Shanxi Academy of Medical Sciences, Shanxi Dayi Hospital, Taiyuan, Shanxi 030032; \\ ${ }^{3}$ Chongqing Key Laboratory of Neurology, Chongqing 40016; ${ }^{4}$ Basic Medicine College \\ of Shanxi Medical University, Taiyuan, Shanxi 030001, P.R. China
}

Received March 4, 2014; Accepted June 20, 2014

DOI: $10.3892 /$ ijmm.2014.1820

\begin{abstract}
Astrocytes are major components of the adult neurogenic niche and play a crucial role in regulating neural stem cell proliferation and differentiation. Following brain injury, astrocytes become reactive and release high-mobility group box 1 (HMGB1), which plays a crucial role in the inflammatory process. However, although it has been reported that HMGB1 promotes neural stem/progenitor cell (NS/PC) proliferation in the developing brain, whether HMGB1 released by reactive astrocytes regulates NS/PC proliferation remains unknown. In this study, we aimed to investigate whether HMGB1 released from reactive astrocytes enhances NS/PC proliferation and to elucidate the possible mechanisms involved in this process. To evaluate the effects of HMGB1 on NS/PC proliferation, NS/PCs were cultured in HMGB1 culture medium and astrocyteconditioned medium with or without reactive astrocyte-derived HMGB1 by RNA interference (RNAi). To explore the possible mechanisms, the HMGB1 receptor for advanced glycation endproducts (RAGE) in the NS/PCs was blocked with antiRAGE antibody, and c-Jun N-terminal protein kinase (JNK) in the NS/PCs was inhibited using the potent JNK inhibitor, SP600125. Our results suggested that HMGB1 released from reactive astrocytes promoted NS/PC proliferation in vitro, and the blockade of RAGE or the inhibition of the JNK signaling pathway in the NS/PCs prevented the HMGB1-induced NS/PC proliferation. Our findings demonstrated that HMGB1 released by reactive astrocytes promoted NS/PC proliferation by binding RAGE and enhancing the phosphorylation of the JNK signaling pathway. These findings support a previously described mechanism of a crosstalk between astrocytes and
\end{abstract}

Correspondence to: Professor Yong Luo, Department of Neurology, The First Affiliated Hospital of Chongqing Medical University, Chongqing Key Laboratory of Neurology, 1 Youyi Road, Yuzhong, Chongqing 40016, P.R. China

E-mail: luoyongneurosci@163.com

Key words: high-mobility group box 1, neural stem/progenitor cell, reactive astrocyte, proliferation, receptor for advanced glycation endproducts, JNK
NS/PCs, and suggest that reactive astrocyte-derived HMGB1 plays an important role in the repair of the central nervous system following brain injury.

\section{Introduction}

Astrocytes are major components of the adult neurogenic niche and play a crucial role in regulating neural stem cell proliferation and differentiation (1-4). Following acute brain injury, such as cerebral ischemia, intracerebral hemorrhage (ICH) or traumatic brain injury, astrocytes become activated and proliferate in the context of brain damage (5-7). Reactive astrocytes secrete numerous pro-inflammatory cytokines which play pathophysiological roles in the subsequent damage.

High-mobility group box 1 (HMGB1) protein is one of the most important pro-inflammatory cytokines secreted by reactive astrocytes $(8,9)$, and mediates a variety of inflammatory responses following insults that include sepsis, pancreatitis and pneumonia (10-12). HMGB1 is a highly conserved non-histone DNA-binding protein that stabilizes nucleosome formation and regulates gene expression by facilitating transcription $(13,14)$. Previous studies have confirmed that following brain injury, HMGB1 acts as an important pro-inflammatory cytokine in mediating inflammation, neuronal apoptosis and tissue damage (15-18). However, emerging data suggest that HMGB1 also appears to have beneficial effects during the recovery stage of brain injury. HMGB1 promotes neurovascular remodeling by recruiting endothelial progenitor cells (EPCs) and increasing EPC proliferation $(7,19,20)$. Roles for HMGB1 in brain development have also been reported, as HMGB1 is associated with neurogenesis, neural progenitor cells survival and proliferation, neurite outgrowth and neuronal differentiation (21-23).

Following brain injury, endogenous neural stem/progenitor cells (NS/PCs) spontaneously proliferate in proximity to the damaged area (24-26). However, the precise mechanisms driving the NS/PC spontaneous proliferation in response to damage remain unknown. Considering that HMGB1 can induce NS/PC proliferation, and is secreted by reactive astrocytes following brain injury, it is highly possible that HMGB1 released by astrocytes may potentiate NS/PC proliferation following brain injury. 
In the present study, we examined the hypothesis that HMGB1 released by astrocytes promotes NS/PC proliferation, and investigated the possible intracellular signaling pathways within the NS/PCs involved in this process. Astrocytes were stimulated with low levels of interleukin (IL)-1 $\beta$ to mimic a reactive phenotype. Subsequently, we used astrocyteconditioned medium (ACM) with or without HMGB1 through the use of RNA interference (RNAi) to evaluate the effects of astrocyte-derived HMGB1 on NS/PC proliferation. We then explored the potential underlying mechanisms in vitro, through the blockade of the receptor for advanced glycation endproducts (RAGE) with an anti-RAGE antibody and by inhibiting the c-Jun $\mathrm{N}$-terminal protein kinase (JNK) signaling pathway with the potent JNK inhibitor, SP600125. The present study provides an experimental basis to explain the potential mechanisms through which NS/PCs spontaneously proliferate following brain injury.

\section{Materials and methods}

Animals. The Ethics Committee of Chongqing Medical University, Chongqing, China approved all protocols for the animal experiments. All procedures were carried out in accordance with the National Institutes of Health (NIH) Guide for the Care and Use of Laboratory Animals (NIH, Bethesda, MD, USA). All animals were provided by the Experimental Animal Center of Chongqing Medical University. Neonatal Sprague-Dawley (SD) rats (1-2 days old) were used for primary astrocyte culture and primary NS/PC culture. All efforts were made to minimize the number of animals used, as well as their suffering.

Study design. In the experiments using ACM, the astrocytes were assigned to 4 groups, and cultured for $24 \mathrm{~h}$ in serum-free $\mathrm{NS} / \mathrm{PC}$ medium with or without IL-1 $\beta(0.1 \mathrm{ng} / \mathrm{ml})$, as previously described by Hayakawa et al (7) (Table I). Four types of conditioned medium were collected and used undiluted in NS/PC proliferation assays: normal ACM (nACM), stimulated ACM (sACM), HMGB1 shRNA interference sACM (HMGB1 shRNA sACM) and control shRNA interference sACM (control shRNA sACM).

In the NS/PC proliferation experiments, the NS/PCs were assigned to 6 groups, and cultured in NS/PC culture medium (vehicle), nACM with IL-1 $\beta(0.1 \mathrm{ng} / \mathrm{ml})$ (control), sACM, HMGB1 shRNA sACM, control shRNA sACM and HMGB1 culture medium (Table I). To eliminate interference by IL-1 $\beta$ and cytokines that were secreted by normal astrocytes, we used nACM containing IL-1 $\beta(0.1 \mathrm{ng} / \mathrm{ml})$ as a control culture (control group). To further confirm that HMGB1 promotes $\mathrm{NS} / \mathrm{PC}$ proliferation, we added $7 \mathrm{ng} / \mathrm{ml}$ recombinant human HMGB1 (approximate to the HMGB1 level in SACM) to serum-free NS/PC medium as an additional control (HMGB1 group). Each group was further divided into 4 subgroups for time course analysis and cultured for 24, 48, 72 and $96 \mathrm{~h}$.

In the analysis of the dual RAGE-JNK signaling pathway, we selected HMGB1 culture medium (NS/PC culture medium containing $7 \mathrm{ng} / \mathrm{ml}$ recombinant human HMGB1). In the RAGE pathway experiments, the NS/PCs were cultured in HMGB1 culture medium in the presence of $\operatorname{IgG}(20 \mu \mathrm{g} / \mathrm{ml})$ or anti-RAGE antibody $(20 \mu \mathrm{g} / \mathrm{ml})$ for $96 \mathrm{~h}$ (Table I). In the JNK signaling pathway experiments, the NS/PCs were cultured in HMGB1 culture medium in the presence of increasing concentrations of the potent JNK inhibitor, SP600125 $(0,1$ and $10 \mu \mathrm{M})$, and serum-free NS/PC culture medium for $96 \mathrm{~h}$ (Table I).

Primary astrocyte culture. Primary astrocytes were prepared from the cortices of 1-2-day-old neonatal SD rats as previously described (7), with some modifications. The cells were grown in high-glucose Dulbecco's modified Eagle's medium (DMEM; Invitrogen, Beijing, China), containing $10 \%$ fetal bovine serum (FBS; Gibco, Life Technologies Ltd., Mount Waverley, Victoria, Australia) and $25 \mu \mathrm{g} / \mathrm{ml}$ penicillin/streptomycin in $95 \%$ air/5\% $\mathrm{CO}_{2}$. To remove non-astrocytic cells, which were mainly microglia, the flasks were shaken overnight at $200 \mathrm{rpm}$. The cells were then trypsinized and seeded into T50 flasks at a density of $6 \times 10^{5}$ cells $/ \mathrm{cm}^{2}$. The cells were cultured for 16-18 days before preparing the ACM and were only used for study when the purity of the astrocytes reached a level of 95\% [verified with Glial fibrillary acidic protein (GFAP) staining].

PrimaryNS/PC culture. NS/PCs were prepared from the cortices of 1-2-day-old neonatal SD rats as previously described (27), with minor modifications. Single cells were plated in uncoated T50 flasks at a density of $1 \times 10^{5}$ cells $/ \mathrm{cm}^{2}$ with NS/PC culture medium composed of DMEM/F12 (Invitrogen) supplemented with $2 \%$ B27 supplement (Invitrogen), $20 \mathrm{ng} / \mathrm{ml}$ epidermal growth factor (EGF; Peprotech, Rocky Hill, NJ, USA) and $20 \mathrm{ng} / \mathrm{ml}$ basic fibroblast growth factor (bFGF; Peprotech). The cultures were maintained for 7 days in a humidified incubator at $37^{\circ} \mathrm{C}$ and $5 \% \mathrm{CO}_{2}$, with half-volume changes of fresh medium every 2-3 days. Neurospheres that formed during the period were dissociated into single cells with $0.125 \%$ trypsin (HyClone, Logan, UT, USA), and the cells were regenerated into neurospheres using NS/PC culture medium. These procedures were repeated 2-3 times before the cells were treated and single-cell suspensions from neurospheres were utilized to examine cell proliferation.

Lentivirus infection of astrocytes. Lentiviral vectors expressing HMGB1 shRNA or control shRNA were obtained from Shanghai Genechem Co. Ltd, Shanghai, China. The sequences of HMGB1 shRNA were as follows: 5'-GATCCCGAAGCA CCCGGATGCTTCTTTCAAGAGAAGAAGCATCCGGG TGCTTTTTTGGAAA-3' (15). Control shRNA consisted of a scrambled sequence that fails to target any known cellular mRNA.

The shRNA-carrying lentiviral vector was used to infect the astrocytes at a multiplicity of infection (MOI) of 10,30 and 50. The lentiviral vectors were prepared according to the transduction protocol for cell cultures provided by Shanghai Genechem. Five days after infection, the cells were collected and the percentage of GFP-positive cells was quantified by fluorescence microscopy. The optimum MOI was found to be 30 , which resulted in a transduction efficiency of $95 \%$. Western blot analysis was then performed to determine the efficiency of HMGB1 knockdown.

Enzyme-linked immunosorbent assay (ELISA). The concentrations of HMGB1 in the nACM, sACM, HMGB1 shRNA sACM and control shRNA sACM were analyzed with a commercially 
Table I. Study design.

\begin{tabular}{|c|c|c|c|}
\hline Experiment & Group & Number & Explanation \\
\hline \multirow{4}{*}{$\begin{array}{l}\text { Astrocyte-conditioned } \\
\text { media (ACM) synthesis } \\
\text { of HMGB1 }\end{array}$} & $\mathrm{nACM}$ & 9 & $\begin{array}{l}\text { Astrocytes were cultured in NS/PC medium for } 24 \mathrm{~h} \text { in the } \\
\text { absence of IL-1 } \beta \text { stimulation }\end{array}$ \\
\hline & sACM & 9 & $\begin{array}{l}\text { Astrocytes were cultured for } 24 \mathrm{~h} \text { in NS/PC medium containing } \\
\mathrm{IL}-1 \beta(0.1 \mathrm{ng} / \mathrm{ml} \text {; Prospec, East Brunswick, NJ, USA })\end{array}$ \\
\hline & HMGB1 shRNA sACM & 9 & $\begin{array}{l}\text { Astrocytes expressing HMGB } 1 \mathrm{shRNA} \text { were cultured for } 24 \mathrm{~h} \\
\text { in NS/PC medium containing } 0.1 \mathrm{ng} / \mathrm{ml} \mathrm{IL-1 \beta}\end{array}$ \\
\hline & Control shRNA sACM & 9 & $\begin{array}{l}\text { Astrocytes expressing control shRNA were cultured for } 24 \mathrm{~h} \text { in } \\
\mathrm{NS} / \mathrm{PC} \text { medium containing } 0.1 \mathrm{ng} / \mathrm{ml} \mathrm{IL-} 1 \beta\end{array}$ \\
\hline \multirow[t]{6}{*}{ NS/PC proliferation } & Vehicle & $9^{\mathrm{a}}$ & NS/PCs were cultured in serum-free NS/PC medium \\
\hline & Control & $9^{\mathrm{a}}$ & NS/PCs were cultured in nACM containing IL- $1 \beta$ \\
\hline & sACM & $9^{\mathrm{a}}$ & NS/PCs were cultured in sACM \\
\hline & HMGB1 shRNA sACM & $9^{\mathrm{a}}$ & $\begin{array}{l}\text { NS/PCs were cultured in sACM from astrocytes expressing } \\
\text { HMGB1 shRNA }\end{array}$ \\
\hline & Control shRNA sACM & $9^{\mathrm{a}}$ & $\begin{array}{l}\text { NS/PCs were cultured in sACM from astrocytes expressing } \\
\text { control shRNA }\end{array}$ \\
\hline & HMGB1 & $9^{\mathrm{a}}$ & $\begin{array}{l}\text { NS/PCs were cultured in serum-free NS/PC medium containing } \\
7 \mathrm{ng} / \mathrm{ml} \text { recombinant human HMGB1 (Prospec) }\end{array}$ \\
\hline \multirow[t]{2}{*}{$\begin{array}{l}\text { RAGE pathway } \\
\text { experiment }\end{array}$} & $\operatorname{IgG}$ & 9 & $\begin{array}{l}\text { NS/PCs were cultured for } 96 \mathrm{~h} \text { in NS/PC culture medium } \\
\text { containing } 7 \mathrm{ng} / \mathrm{ml} \mathrm{HMGB} 1 \text { and } 20 \mu \mathrm{g} / \mathrm{ml} \text { control } \mathrm{IgG} \\
\text { (Beyotime Institute of Biotechnology, Wuhan, China) }\end{array}$ \\
\hline & Anti-RAGE & 9 & $\begin{array}{l}\text { NS/PCs were cultured for } 96 \mathrm{~h} \text { in NS/PC culture medium } \\
\text { containing } 7 \mathrm{ng} / \mathrm{ml} \mathrm{HMGB} 1 \text { and } 20 \mu \mathrm{g} / \mathrm{ml} \text { anti-RAGE antibody } \\
\text { (Santa Cruz Biotechnology, Santa Cruz, CA, USA) }\end{array}$ \\
\hline \multirow[t]{4}{*}{ JNK pathway analysis } & Vehicle & 9 & NS/PCs were cultured for $96 \mathrm{~h}$ in serum-free NS/PC medium \\
\hline & $0 \mu \mathrm{M}$ & 9 & $\begin{array}{l}\text { NS/PCs were cultured for } 96 \mathrm{~h} \text { in NS/PC culture medium } \\
\text { containing } 7 \mathrm{ng} / \mathrm{ml} \mathrm{HMGB} 1\end{array}$ \\
\hline & $1 \mu \mathrm{M}$ & 9 & $\begin{array}{l}\text { NS/PCs were cultured for } 96 \mathrm{~h} \text { in NS/PC culture medium } \\
\text { containing } 7 \mathrm{ng} / \mathrm{ml} \mathrm{HMGB} 1 \text { and } 1 \mu \mathrm{M} \mathrm{SP} 600125\end{array}$ \\
\hline & $10 \mu \mathrm{M}$ & 9 & $\begin{array}{l}\text { NS/PCs were cultured for } 96 \mathrm{~h} \text { in NS/PC culture medium } \\
\text { containing } 7 \mathrm{ng} / \mathrm{ml} \mathrm{HMGB} 1 \text { and } 10 \mu \mathrm{M} \mathrm{SP} 600125\end{array}$ \\
\hline
\end{tabular}

andicates 9 samples at each time point $(24,48,72$ and $96 \mathrm{~h})$.

available ELISA kit according to the manufacturer's instructions (Cat. no. CSB-E08224r/96T; Cusabio Biotech Co., Ltd., Wuhan, China).

CCK-8 proliferation assays for NS/PCs. The CCK-8 (Cat. no. C0038; Beyotime Institute of Biotechnology, Shanghai, China) proliferation assay was used to determine the rate of NS/PC proliferation. Single NS/PCs were seeded into 96-well plates (10,000 cells/well in $100 \mu \mathrm{l}$ medium) with corresponding medium. CCK-8 solution $(10 \mu \mathrm{l})$ was added to the cell culture medium and following incubation for $2 \mathrm{~h}$ at $37^{\circ} \mathrm{C}$, the absorbance of the culture medium was determined at a wavelength of $450 \mathrm{~nm}$ with a reference wavelength of $630 \mathrm{~nm}$ by using a multiskan spectrum scanning spectrophotometer (Thermo Labsystems, Vantaa, Finland). Using these procedures, a good linear correlation was obtained between the absorbance and viable cell number. Each experiment was performed in triplicate and the results were collected as the average of at least 3 independent experiments.
NS/PC cell cycle analysis. The effects of the different culture media on cell cycle distribution were measured by flow cytometry, as previously described (28). The NS/PCs were dissociated into single-cell suspensions, and fixed in $70 \%$ ice-cold ethanol overnight at $4^{\circ} \mathrm{C}$. The fixed cells were stained with propidium iodide $(50 \mu \mathrm{g} / \mathrm{ml}$; BD Biosciences Franklin Lakes, NJ, USA), and $50 \mu \mathrm{g} / \mathrm{ml}$ RNAse A (BD Biosciences) at $37^{\circ} \mathrm{C}$ for $30 \mathrm{~min}$ in the dark, and subsequently analyzed using a flow cytometer (FACSVantage SE; BD Biosciences). The changes in cell cycle distribution were determined by calculating the proliferation index $(\mathrm{PI})$. The following formula was used: $\mathrm{PI}=(\mathrm{S}+\mathrm{G} 2 / \mathrm{M}) /$ (G0/G1 $+\mathrm{S}+\mathrm{G} 2 / \mathrm{M})$, as previously described (28).

Double-immunofluoresence labeling. The cultured astrocytes and neurospheres were washed and fixed with $4 \%$ paraformaldehyde at $4^{\circ} \mathrm{C}$ for $30 \mathrm{~min}$. The astrocytes were incubated overnight at $4^{\circ} \mathrm{C}$ with the following primary antibodies: mouse anti-GFAP (1:300; Cell Signaling Technology, Danvers, MA, USA) and rabbit anti-HMGB1 antibodies (1:100; Abcam, 
Cambridge, MA, USA). Neurospheres were incubated overnight at $4^{\circ} \mathrm{C}$ with the following primary antibodies: rabbit anti-nestin (1:50; Proteintech, Wuhan, China) and mouse anti-Sox-2 antibodies (1:100; Cell Signaling Technology). The cells were then incubated with secondary antibodies, including goat anti-mouse Alexa Fluor 647 (1:200; Beyotime Institute of Biotechnology), goat anti-rabbit TRITC (1:100) and goat anti-mouse FITC (1:100) (all from Beijing Ding Guo Changsheng Biotech Co., Ltd., Beijing, China) for $1 \mathrm{~h}$ at $37^{\circ} \mathrm{C}$ in the dark. Finally, the cells were examined by laser-scanning confocal microscopy on an Olympus IX70 inverted microscope (Olympus, Tokyo, Japan) equipped with a FluoView FVX confocal scan head (Leica Microsystems GmbH, Wetzlar, Germany).

Western blot analysis. Total protein derived from the astrocytes and NS/PCs was harvested with RIPA lysis buffer containing a protease and phosphatase inhibitor cocktail (KeyGen Biotech Co., Ltd., Nanjing, China). The protein concentrations were measured using the Bradford method (Beyotime Institute of Biotechnology). The protein samples $(50 \mu \mathrm{g})$ were fractionated by $10 \%$ SDS-polyacrylamide gel electrophoresis, electroblotted onto a polyvinylidene difluoride (PVDF; Millipore, Billerica, MA, USA) membrane and immunoblotted with primary antibodies, including: rabbit anti-HMGB1 $(1: 1,000)$, rabbit anti-phosphorylated JNK (anti-p-JNK, 1:1,000; Cell Signaling Technology), rabbit anti-JNK (1:1,000; Cell Signaling Technology) and mouse anti- $\beta$-actin anbitody (1:1000, Beijing Ding Guo Changsheng Biotech) as an internal control. A Bio-Rad apparatus (Bio-Rad Laboratories, Richmond, CA, USA) and Quantity One software version 4.6.2 (Bio-Rad Laboratories) were used to scan the immunoblots for semi-quantitative analysis.

Statistical analysis. The statistical software program SPSS 18.0 for Windows (SPSS Inc., Chicago, IL,USA) was used to conduct statistical analysis. All data are presented as the means \pm standard deviation (SD). Statistical analysis was performed by one-way ANOVA, and when $\alpha$ values were at $\mathrm{P}<0.05$, the Bonferroni test was used for homogeneity of variance, and the Tamhane test was used for heterogeneity of variance. Values of $\mathrm{P}<0.05$ were considered to indicate statistically significant differences.

\section{Results}

$I L-1 \beta$-stimulated astrocytes can release $H M G B 1$, and $R N A i$ successfully suppresses the release of HMGB1. To determine the effects of IL-1 $\beta$ and RNAi on HMGB1 secretion, we examined the HMGB1 concentrations in the ACM. HMGB1 protein expression was barely detectable in the $\mathrm{nACM}(0.060 \pm 0.078 \mathrm{ng} /$ $\mathrm{ml})$. After $24 \mathrm{~h}$ of stimulation with IL-1 $\beta$, sACM showed a clear accumulation of soluble HMGB1 $(6.929 \pm 0.630 \mathrm{ng} / \mathrm{ml})$. Compared with that in SACM, the HMGB1 concentration in the HMGB1 shRNA sACM $(0.707 \pm 0.164 \mathrm{ng} / \mathrm{ml})$ was markedly decreased by RNAi (Fig. 1). Additionally, there was almost no effect on the HMGB1 concentration in the control shRNA sACM $(6.917 \pm 0.586 \mathrm{ng} / \mathrm{ml})$, compared with that in the SACM (Fig. 1).

$I L-1 \beta$ upregulates $H M G B 1$ protein expression in astrocytes, and RNAi successfully suppresses the expression of HMGB1.

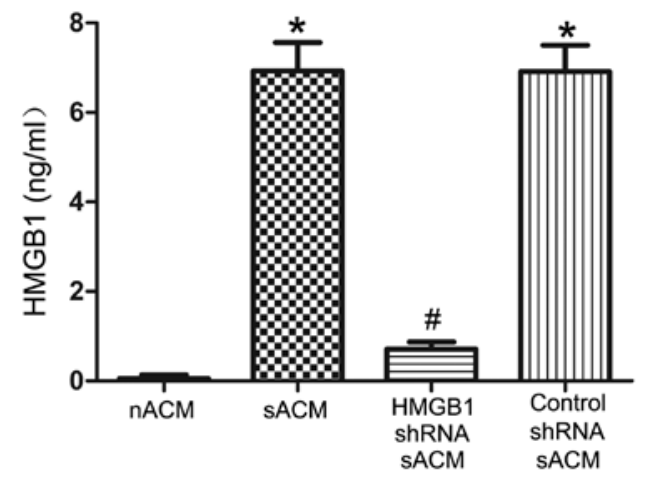

Figure 1. Release of HMGB1 from IL-1 $\beta$-stimulated astrocytes. ELISA confirmed that HMGB1 was undetectable in normal astrocyte-conditioned medium (nACM). IL-1 $\beta(0.1 \mathrm{ng} / \mathrm{ml})$ stimulation caused astrocytes to release HMGB1 into stimulted astrocyte-conditioned medium (sACM). HMGB1 shRNA attenuated the release of HMGB1 into the ACM (HMGB1 shRNA SACM). A control shRNA (control shRNA sACM) had no effect on HMGB1 release into IL-1 $\beta$-stimulated ACM. ${ }^{*} \mathrm{P}<0.001$ vs. $\mathrm{nACM}$; ${ }^{\text {"}} \mathrm{P}<0.001$ vs. sACM.

To determine the effects of IL-1 $\beta$ and RNAi on intracellular HMGB1 expression, we examined the HMGB1 protein levels in cell lysates obtained from astrocytes and double-immunofluorescence-stained astrocytes. Western blots of astrocyte lysates (Fig. 2A) and the double-immunofluorescence staining of astrocytes (HMGB1/astrocytes red/blue; Fig. 2B) confirmed that endogenous HMGB1 protein was indeed upregulated in the IL-1 $\beta$-stimulated astrocytes compared to the normal astrocytes. HMGB1 shRNA effectively suppressed HMGB1 protein expression in the IL-1 $\beta$-stimulated astrocytes. Control shRNA had no effect on HMGB1 expression in the IL- $1 \beta$-stimulated astrocytes, compared to that of the IL-1 $\beta$-stimulated astrocytes.

Neurospheres cultured in vitro were identified with the representative markers, nestin and SRY-re lated HMG-box gene 2 (Sox-2). To characterize the cell population, we performed double-labelling experiments with markers of undifferentiated neural stem/progenitor cells, including the intermediate neurofilament protein, nestin, and Sox-2, a transcription factor expressed in undifferentiated stem/progenitor cell nuclei (29) (Fig. 3A). Infant rat cortex-derived neurospheres grown in the presence of EGF and bFGF are mainly composed of nestin (red) and Sox-2 (green)-positive cells. The results of doubleimmunolabelling experiments and the light microscopy of neurospheres (Fig. 3B) suggested that they are composed of undifferentiated NS/PCs.

HMGB1 released by astrocytes promotes NS/PC proliferation in vitro. To determine whether HMGB1 released from astrocytes affects NS/PC proliferation, we performed an in vitro CCK- 8 assay. The absorbance of CCK- 8 in the NS/PC proliferation experiments is shown in Table II. The results indicatedd that there were no differences in the proliferation rates among each of the groups at 24 or $48 \mathrm{~h}(\mathrm{P}>0.05)$. However, as shown in Fig. 4, after 72 and $96 \mathrm{~h}$ of exposure, the NS/PC proliferation rates in the SACM group, control shRNA sACM group and HMGB1 group increased significantly compared to the vehicle control group. Compared to the sACM group, the NS/PC proliferation rates in the HMGB1 shRNA sACM 
Table II. Effects of astrocyte-conditioned medium on the CCK-8 absorbance of NS/PCs ( $n=9$, values indicate the means \pm SD).

\begin{tabular}{|c|c|c|c|c|c|c|}
\hline $\begin{array}{l}\text { Time } \\
\text { point }(\mathrm{h})\end{array}$ & Vehicle & Control & sACM & sACM HMGB1 shRNA & sACM control shRNA & HMGB1 \\
\hline 24 & $0.197 \pm 0.036$ & $0.196 \pm 0.033$ & $0.175 \pm 0.027$ & $0.196 \pm 0.032$ & $0.179 \pm 0.026$ & $0.180 \pm 0.033$ \\
\hline 48 & $0.294 \pm 0.041$ & $0.298 \pm 0.048$ & $0.319 \pm 0.050$ & $0.313 \pm 0.039$ & $0.310 \pm 0.046$ & $0.312 \pm 0.031$ \\
\hline 72 & $0.404 \pm 0.047$ & $0.413 \pm 0.049$ & $0.490 \pm 0.041$ & $0.423 \pm 0.030$ & $0.487 \pm 0.041$ & $0.479 \pm 0.045$ \\
\hline 96 & $0.765 \pm 0.059$ & $0.745 \pm 0.071$ & $0.966 \pm 0.056$ & $0.848 \pm 0.051$ & $0.980 \pm 0.053$ & $0.984 \pm 0.073$ \\
\hline
\end{tabular}
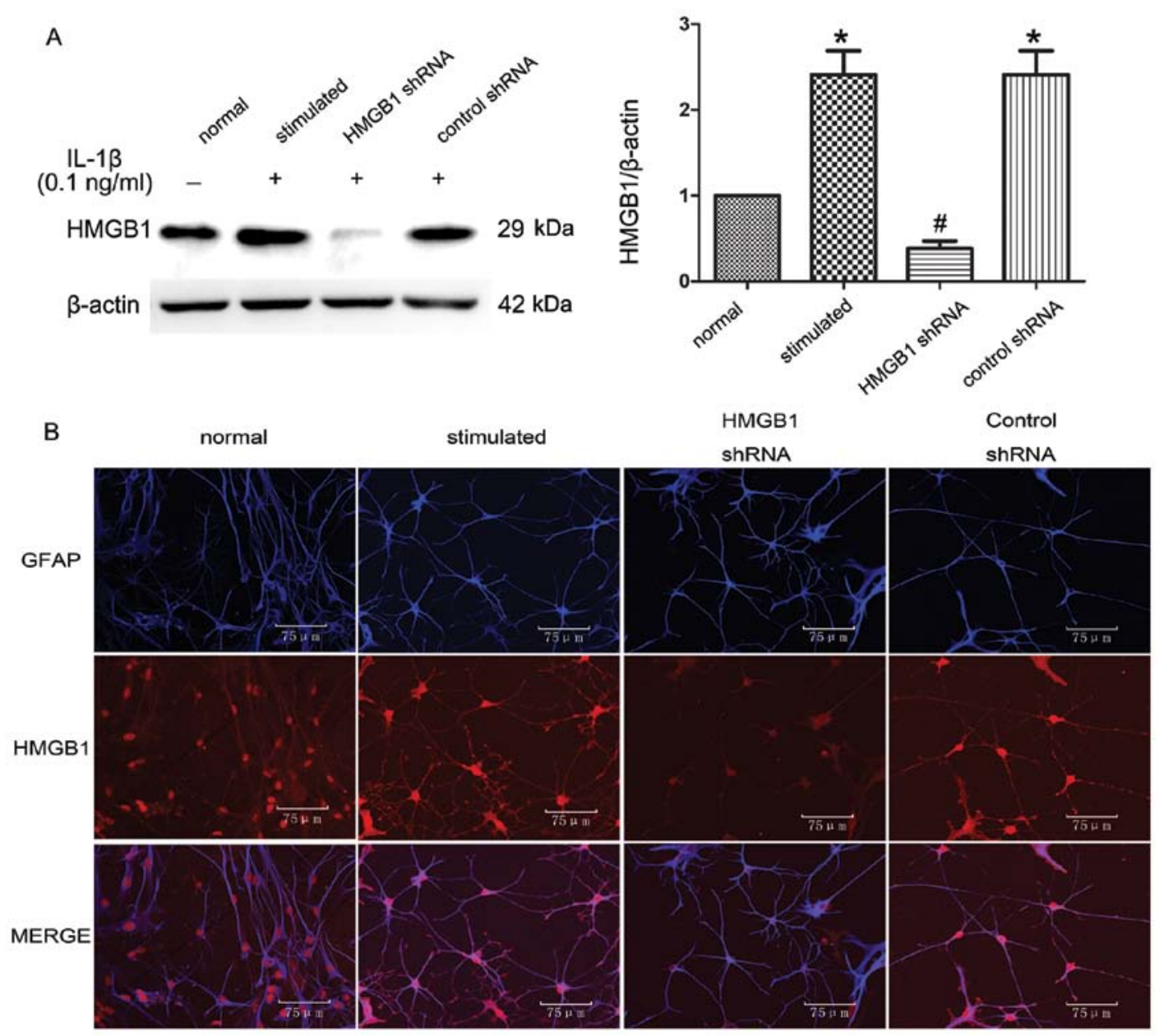

Figure 2. (A) Western blot analysis and (B) double-immunofluorescence labeling of HMGB1 expression in IL-1 $\beta$-stimulated astrocytes. (A) HMGB1 protein expression was upregulated in IL-1 $\beta$-stimulated astrocytes. HMGB1 shRNA specifically suppressed HMGB1 protein expression in IL-1 $\beta$-stimulated astrocytes, as a control shRNA had no effect on HMGB1 protein expression in IL-1 $\beta$-stimulated astrocytes. ${ }^{\prime \prime} \mathrm{P}<0.001$ vs. unstimulated astrocytes; ${ }^{\prime \prime} \mathrm{P}<0.001$ vs. stimulated astrocytes. (B) Double-immunofluorescence labeling of astrocytes for GFAP (blue) and HMGB1 (red). HMGB1 expression was upregulated in IL-1 $\beta$-stimulated GFAP ${ }^{+}$astrocytes. HMGB1 shRNA successfully suppressed HMGB1 expression in IL-1 $\beta$-stimulated GFAP ${ }^{+}$astrocytes. There was no difference in HMGB1 expression between IL-1 $\beta$-stimulated $\mathrm{GFAP}^{+}$astrocytes and IL-1 $\beta$-stimulated control shRNA astrocytes.
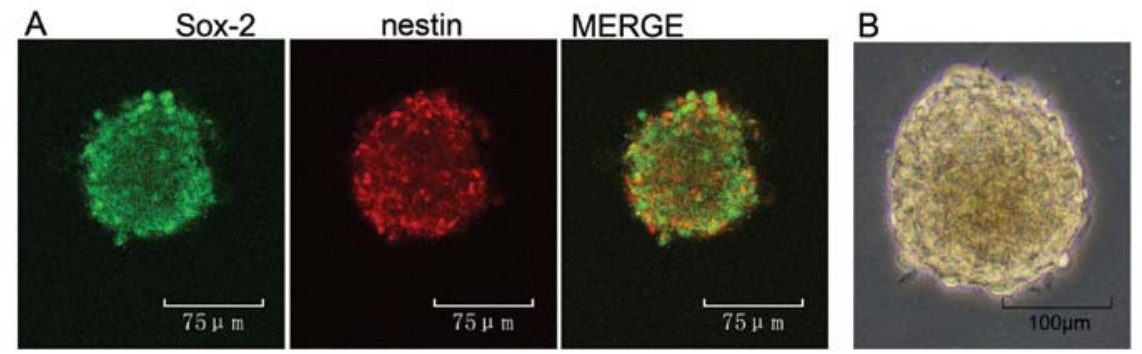

Figure 3. Identification of neurospheres. (A) Neurospheres were identified by the representative markers, Sox-2 (green) and nestin (red). (B) A representative neurosphere observed under a light microscope. 
Table III. Effects of astrocyte-conditioned medium on the proliferation index (PI) of NS/PCs (n=9, values indicate the means \pm SD)

\begin{tabular}{lccccr}
\hline $\begin{array}{l}\text { Time } \\
\text { point }(\mathrm{h})\end{array}$ & Vehicle & Control & sACM & sACM HMGB1 shRNA & sACM control shRNA \\
\hline $24 \mathrm{~h}$ & $17.87 \pm 0.92$ & $17.87 \pm 0.86$ & $17.69 \pm 0.97$ & $17.72 \pm 0.96$ & $17.84 \pm 1.09$ \\
$48 \mathrm{~h}$ & $18.95 \pm 0.96$ & $18.58 \pm 1.00$ & $18.81 \pm 0.96$ & $19.09 \pm 1.07$ & $18.65 \pm 1.07$ \\
$72 \mathrm{~h}$ & $19.45 \pm 1.12$ & $19.48 \pm 0.97$ & $22.07 \pm 1.61$ & $20.06 \pm 1.00$ & $21.95 \pm 1.69$ \\
$96 \mathrm{~h}$ & $19.89 \pm 1.27$ & $19.48 \pm 0.96$ & $27.04 \pm 1.00$ & $21.13 \pm 1.09$ & $27.38 \pm 0.63$ \\
\hline
\end{tabular}

$\mathrm{PI}=(\mathrm{S}+\mathrm{G} 2 / \mathrm{M}) /(\mathrm{G} 0 / \mathrm{G} 1+\mathrm{S}+\mathrm{G} 2 / \mathrm{M})$.
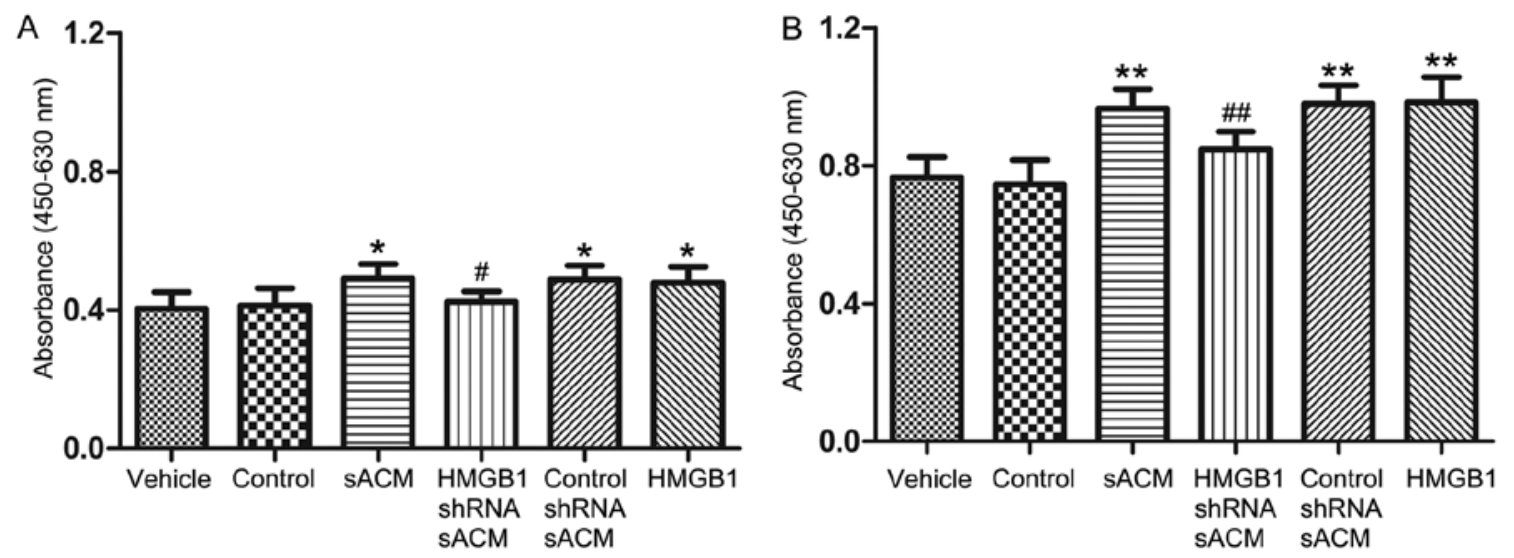

Figure 4. The effects of astrocyte-derived HMGB1 on NS/PC proliferation were measured by the CCK-8 assay. Relative absorbance values of viable NS/PCs were measured by CCK-8 assay at (A) $72 \mathrm{~h}$ and (B) $96 \mathrm{~h}$. The proliferation of NS/PCs in sACM, control shRNA sACM and HMGB1 culture media was increased following 72 and $96 \mathrm{~h}$ of exposure. Compared with the sACM, the proliferation of NS/PCs in HMGB1 shRNA sACM significantly decreased after 72 and $96 \mathrm{~h}$. There were no intra-time point differences in NS/PC proliferation between the vehicle (NS/PC culture medium only) and control (normal ACM with IL-1 $\beta$ ) groups at either the 72 - or $96-\mathrm{h}$ timepoint. ${ }^{*} \mathrm{P}<0.01,{ }^{* *} \mathrm{P}<0.001$ vs. vehicle group; ${ }^{*} \mathrm{P}<0.05,{ }^{\# \#} \mathrm{P}<0.01$ vs. sACM group.

group exhibited a statistically significant decrease at 72 and $96 \mathrm{~h}$, while the NS/PC proliferation rates in the control group showed no statistical differences at 72 or $96 \mathrm{~h}$ compared to the vehicle group $(\mathrm{P}>0.05)$.

$H M G B 1$ released by astrocytes promotes NS/PC proliferation in vitro by regulating the cell cycle. To further investigate the effects of astrocyte-derived HMGB1 on NS/PC proliferation, we analyzed the cell cycle of NS/PCs by flow cytometry in a time course experiment using the different culture media. The PI was selected to reflect the proliferative ability of the NS/PCs, using the following formula: $\mathrm{PI}=(\mathrm{S}+\mathrm{G} 2 / \mathrm{M}) /(\mathrm{G} 0 /$ $\mathrm{G} 1+\mathrm{S}+\mathrm{G} 2 / \mathrm{M})$, as previously described (28). The PI in the NS/PC proliferation experiments is shown in Table III. There was no statistically significant difference in the PI following culture for 24 or $48 \mathrm{~h}(\mathrm{P}>0.05)$ with the different culture media. Compared with the PI of the vehicle group, the PI of the sACM group, control shRNA sACM group and HMGB1 group showed a significant increase after 72 and 96 h (Fig. 5). Compared with the sACM group, the PI of the HMGB1 shRNA sACM group exhibited a statistically significant decrease after 72 and 96 h (Fig. 5). There was no significant difference in the PI between the vehicle group and the control group at all 4 time points $(\mathrm{P}>0.05)$.
HMGB1 promotes NS/PC proliferation through the activation of the RAGE-dependent JNK signaling pathway. To explore the mechanisms behind HMGB1-mediated NS/PC proliferation, we investigated the RAGE receptor pathway, a critical receptor for HMGB1, and the JNK signaling pathway, a signaling pathway involved in HMGB1-RAGE signaling (30). First, we performed an in vitro CCK-8 assay in which the NS/ PCs were placed in HMGB1 culture medium for $96 \mathrm{~h}$ with or without anti-RAGE antibodies. As shown in Fig. 6A, the NS/ $\mathrm{PC}$ proliferation rates in the anti-RAGE group (absorbance, $0.581 \pm 0.068)$ decreased significantly compared with the $\mathrm{IgG}$ group (absorbance, 0.919 \pm 0.069 ). Correspondingly, the p-JNK protein levels were significantly attenuated in the anti-RAGE group compared to the IgG group (Fig. 6B).

To further determine the effects of the JNK signaling pathway on HMGB1-mediated NS/PC proliferation, the $\mathrm{NS} / \mathrm{PCs}$ were incubated for $96 \mathrm{~h}$ in HMGB1 culture medium with various concentrations of the JNK inhibitor, SP600125. As shown in Fig. 6C, compared with the vehicle group, the p-JNK levels in the $0 \mu \mathrm{M}$ group and $1 \mu \mathrm{M}$ group were markedly increased, while the p-JNK levels significantly decreased in the $10 \mu \mathrm{M}$ group compared to the $0 \mu \mathrm{M}$ group. Correspondingly, the NS/PC proliferation rates significantly increased in the $0 \mu \mathrm{M}$ group (absorbance, $0.920 \pm 0.078$ ) and 


\section{A Cell cycle kinetics of NS/PCs at $72 \mathrm{~h}$}
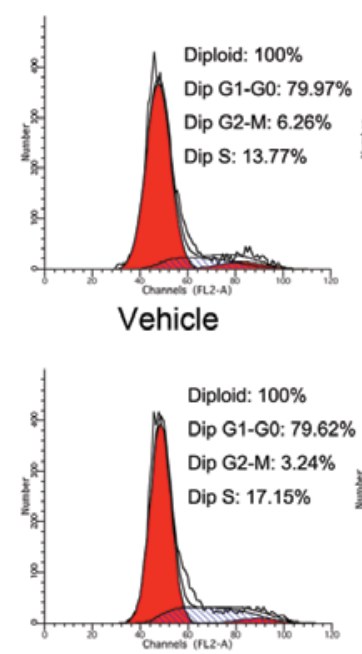

HMGB1 shRNA sACM

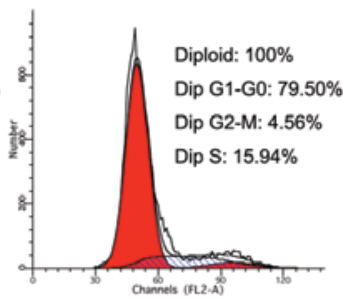

Control

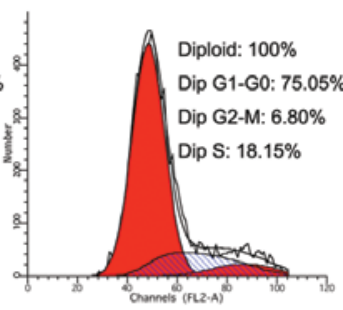

Control shRNA sACM
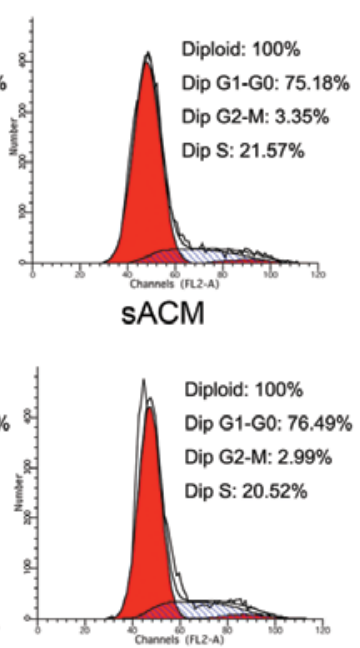

HMGB1

\section{B Cell cycle kinetics of NS/PCs at $96 \mathrm{~h}$}

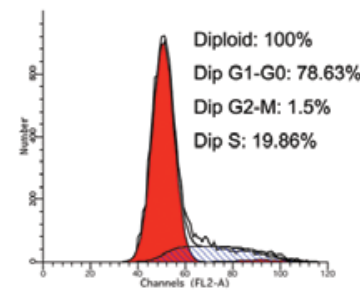

Vehicle

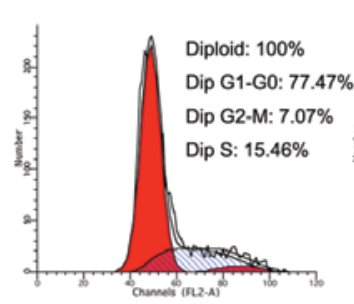

HMGB1 shRNA sACM

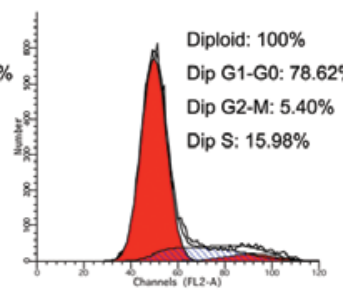

Control

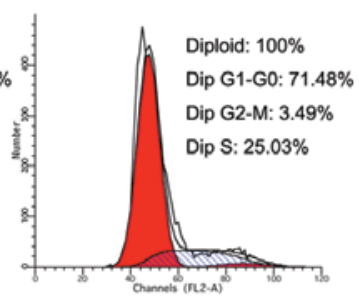

SACM

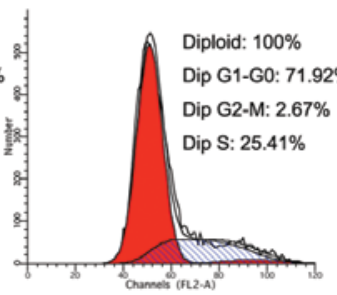

Control shRNA sACM

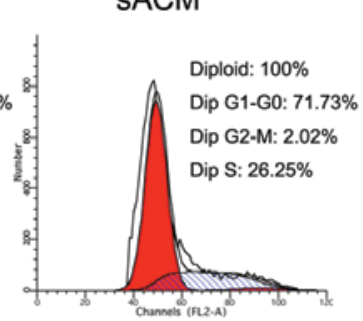

HMGB1
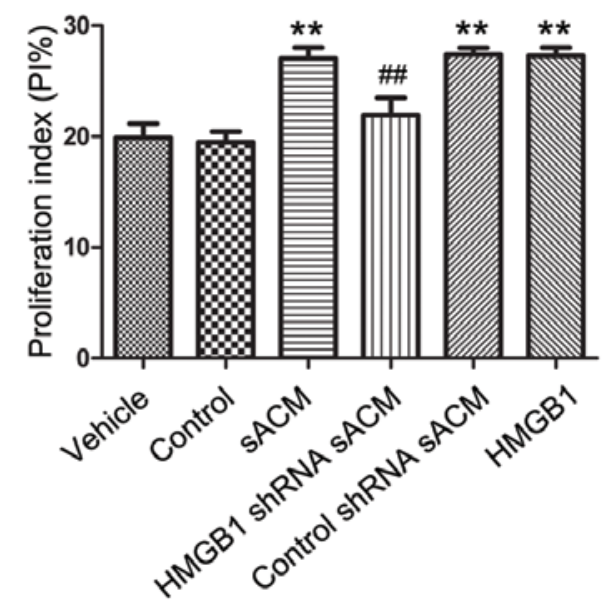

Figure 5. Astrocyte-derived HMGB1 on NS/PC proliferation was measured by analyzing the cell cycle. The cell cycle kinetics of NS/PCs were analyzed by flow cytometry at (A) $72 \mathrm{~h}$ and (B) $96 \mathrm{~h}$. The proliferative capacity of NS/PCs was described as the proliferation index $(\mathrm{PI}): \mathrm{PI}=(\mathrm{S}+\mathrm{G} 2 / \mathrm{M}) /(\mathrm{G} 0 / \mathrm{G} 1+\mathrm{S}+\mathrm{G} 2 / \mathrm{M})$. The PIs of the sACM group, control shRNA sACM group and HMGB1 group were increased after 72 and $96 \mathrm{~h}$. Compared with the sACM group, the PI of the HMGB1 shRNA sACM group was significantly decreased after 72 and $96 \mathrm{~h}$. There were no intra-time point differences in PI between vehicle (NS/PC culture medium only) and control (normal ACM with IL-1 $\beta$ ) groups at either the 72 or $96-\mathrm{h}$ time point. ${ }^{*} \mathrm{P}<0.01,{ }^{* *} \mathrm{P}<0.001$ vs. vehicle group; ${ }^{~} \mathrm{P}<0.05$, ${ }^{\# \#} \mathrm{P}<0.001$ vs. sACM group.

$1 \mu \mathrm{M}$ group (absorbance, $0.914 \pm 0.047$ ), compared with the vehicle group (absorbance, $0.723 \pm 0.055$ ) (Fig. 6D), and the $\mathrm{NS} / \mathrm{PC}$ proliferation rates were significantly decreased in the $10 \mu \mathrm{M}$ group (absorbance, $0.700 \pm 0.045$ ) relative to the $0 \mu \mathrm{M}$ group.

\section{Discussion}

In the present study, the CCK-8 proliferation assay and PI were selected to reflect the proliferative capacity of the NS/ PCs. NS/PCs treated with IL-1 $\beta$-stimulated ACM (sACM) displayed increased proliferation rates in vitro. When endog- enous HMGB1 in the IL-1 $\beta$-stimulated ACM was suppressed using shRNA, the ability of the sACM to evoke in vitro NS/ PC proliferation was decreased. The addition of exogenous HMGB1 to the NS/PC cultures also increased the NS/PC proliferation rates in vitro. Furthermore, treatment of the HMGB1-induced NS/PCs with an anti-RAGE antibody significantly decreased both the NS/PC proliferation rates and p-JNK protein levels. Finally, treatment of the HMGB1induced NS/PCs with various concentrations of the JNK inhibitor, SP600125, revealed that p-JNK levels decreased concomitantly with the proliferation rates of NS/PCs. These results suggest that astrocyte-derived HMGB1 promotes NS/ 


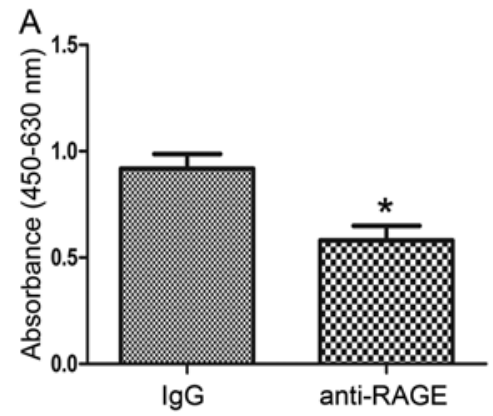

C

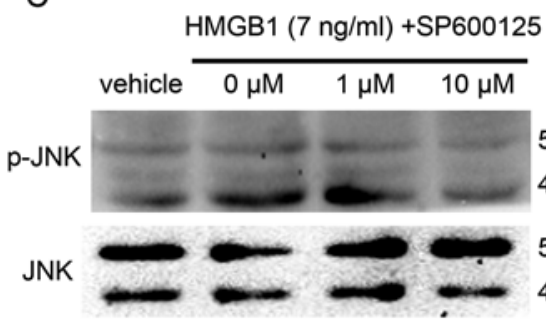

B
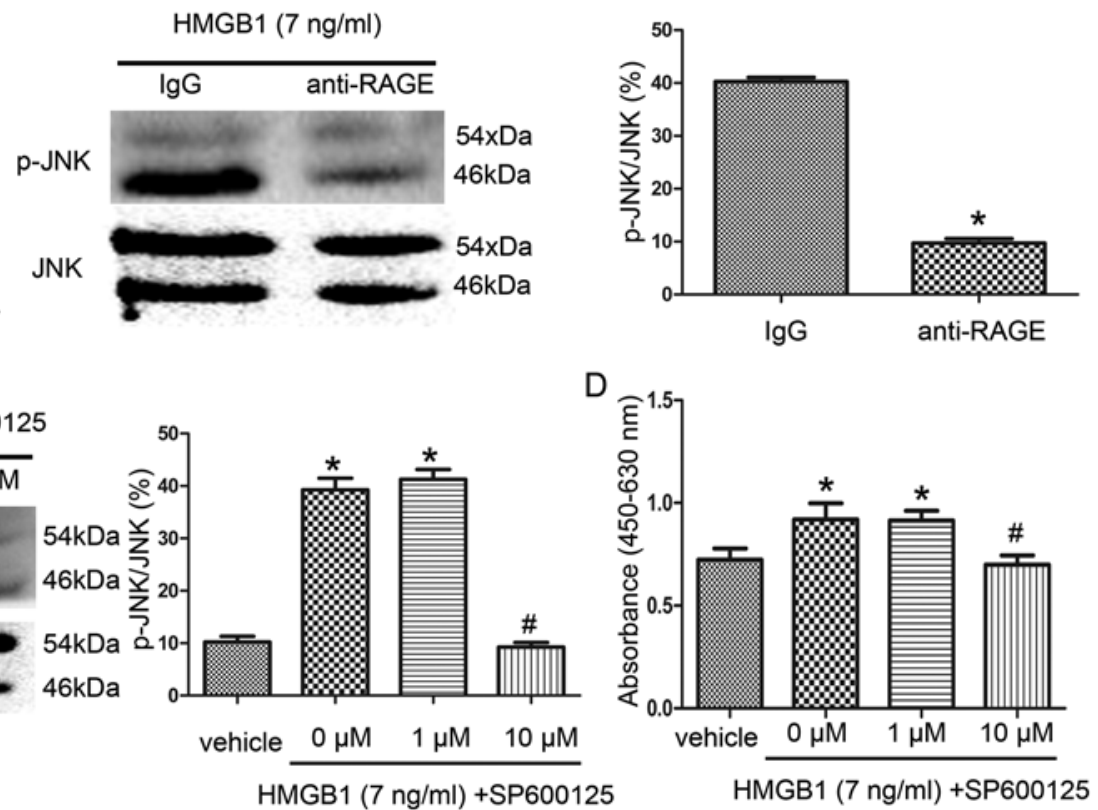

Figure 6. Analysis of signaling pathways involved in HMGB1-mediated NS/PC proliferation by CCK-8 assay (A and D) and western blot analysis (B and C). $\mathrm{NS} / \mathrm{PCs}$ were cultured for $96 \mathrm{~h}$ in NS/PC culture medium containing $7 \mathrm{ng} / \mathrm{ml}$ HMGB1. (A) Compared to an IgG control, blockade of RAGE with an anti-RAGE antibody significantly inhibited HMGB1-induced NS/PC proliferation. " P<0.001. (B) Western blot analysis showed that the blockade of RAGE reduced p-JNK levels in NS/PCs. "P<0.001. (C) Exposure of NS/PCs to HMGB1-containing culture medium rapidly increased p-JNK levels in NS/PCs; the JNK inhibitor, SP600125, reduced JNK phosphorylation in the NS/PCs. ${ }^{*} \mathrm{P}<0.001$ vs. vehicle group; ${ }^{\#} \mathrm{P}<0.001$ vs. $0 \mu \mathrm{M}$ group. (D) SP600125 (10 $\mu \mathrm{M}$ ) significantly reduced the ability of HMGB1 to enhance NS/PC proliferation. ${ }^{*} \mathrm{P}<0.001$ vs. vehicle group; ${ }^{\#} \mathrm{P}<0.001$ vs. $0 \mu \mathrm{M}$ group.

PC proliferation in vitro, and that HMGB1-stimulated NS/PC proliferation may be mediated through the RAGE-dependent JNK signaling pathway.

As major components of the neurogenic niche, astrocytes play a crucial role in regulating neural stem cell proliferation and differentiation (1-4). However, following injury to the central nervous system, traditional thinking presumes that astrocytes are activated to form glial scars and promote inflammation, which is detrimental to neuronal recovery (31-34). However, emerging data suggest that the role of reactive astrocytes may be more complex than previously appreciated. It has been reported that reactive astrocytes expressing HMGB1 in the peri-infarct cortex may promote neurovascular remodeling and functional recovery after stroke, suggesting that these cells may in fact also possess beneficial effects $(7,19)$. In the present study, we found that reactive ACM containing low levels of HMGB1 stimulated NS/PC proliferation in vitro. HMGB1 is a non-histone DNA-binding protein which stabilizes nucleosome formation and regulates gene expression $(13,14)$. HMGB1 is also a multifunctional molecule that can act as a vital pro-inflammatory cytokine to trigger inflammation by stimulating the secretion of pro-inflammatory cytokines and chemokines, including tumor necrosis factor- $\alpha$ (TNF- $\alpha$ ), IL-2, IL-6 and other cytokines $(13,14)$. Hence, studies have focused on blocking HMGB1 signaling in order to reduce damage following brain injury $(15,16)$.

In contrast to these negative effects, a growing body of literature indicates that HMGB1 may also have the potential to promote tissue regeneration. It has been reported that following a tissue lesion, HMGB1 can stimulate certain populations of cells to proliferate, such as mesoangioblasts (vessel-associated stem cells), myocardial cells and EPCs $(7,35,36)$. It has also been reported that low levels of exogenous HMGB1 (1-10 ng/ $\mathrm{ml})$ promote EPC and neuronal precursor cell (NPC) proliferation in vitro $(7,29)$. Previous studies have suggested that stimulated astrocytes can produce and release HMGB1 into the extracellular medium $(8,9)$, and the release of HMGB1 from astrocytes has been shown to increase EPC proliferation both in vitro and in vivo (7). In this context, HMGB1 may also provide a potential missing link between reactive astrocytes and NS/PC proliferation, and the results presented in this study are consistent with this hypothesis. Firstly, when the expression of astrocytic HMGB1 was inhibited using shRNA, the ability of the sACM to promote NS/PC proliferation was blocked. Secondly, when exogenous HMGB1 was added to the NS/PC culture medium, the HMGB1 culture medium also enhanced the NS/PC proliferation rates. Taken together, these results suggest that it was HMGB1 in the sACM that increased $\mathrm{NS} / \mathrm{PC}$ proliferation in vitro.

These experiments identified that astrocytic HMGB1 had a relatively modest and slow effect on NS/PC proliferation in vitro. Previous studies have identified that concentrations of HMGB1 ranging from 1 to $10 \mathrm{ng} / \mathrm{ml}$ significantly increase neural progenitor cell proliferation in vitro (29). Hayakawa et al (7) used multiple doses of HMGB1 and showed biphasic effects on proliferation, where low levels of HMGB1 (1-10 ng/ml) increased the proliferation rates of EPCs, but higher concentrations $(100-1,000 \mathrm{ng} / \mathrm{ml})$ appeared to have no effect, suggesting that high concentrations of HMGB1 may be deleterious by promoting endothelial inflammation and inducing neurotoxicity. In the experiments presented in this study, the concentration of HMGB1 in the $\mathrm{sACM}(\sim 7 \mathrm{ng} / \mathrm{ml})$ 
was within the limits of low levels of HMGB1 (1-10 ng/ml). We further identified that low levels of endogenous HMGB1 secreted from astrocytes can also increase NS/PC proliferation in vitro. In our experiments, the NS/PC proliferation rates exhibited a relatively stationary phase for up to $48 \mathrm{~h}$ and a statistically significant increased rate from 72 to $96 \mathrm{~h}$. The time scales are consistent with previous findings reported by Meneghini et al (29) that HMGB1 produced a relatively stationary phase up to $48 \mathrm{~h}$ and a statistically significant increase at $72 \mathrm{~h}$. In our experiments, we stimulated astrocytes with low levels of IL-1 $\beta$ in vitro to mimic the in vivo inflammatory microenviroment. Since others have reported that as little as $0.8 \mathrm{ng} / \mathrm{ml} \mathrm{IL-1} \beta$ inhibits the proliferation of NPCs (37), and that astrocytes may release certain cytokines that affect NPC proliferation, we used a control group in which nACM was supplemented with IL-1 $\beta(0.1 \mathrm{ng} / \mathrm{ml})$. We found that there was no effect on the proliferation of NS/PCs. In these experiments, we used ACM, but not NS/PC-astrocyte co-cultures; therefore, it is clear that molecules secreted by astrocytes, but not cell surface molecules on astrocytes, regulate NS/PC proliferation. All the findings in these in vitro experiments demonstrate that HMGB1 released from reactive astrocytes promotes the proliferation of NS/PCs.

We also provided evidence of the presence of a functional RAGE-JNK axis in HMGB1-mediated NS/PC proliferation. As a receptor of HMGB1, RAGE plays an important role in regulating stem cell proliferation and differentiation. Meneghini et al (29) reported that HMGB1 interacted with RAGE and stimulated both proliferation and neuronal differentiation of subventricular zone (SVZ)-derived NS/PCs in vitro. Additionally, Kim et al (38) demonstrated that HMGB1 may interact with RAGE and have important functions during the neuronal differentiation of NT2/D1 cells. In our in vitro NS/ PC culture system, after blocking the activation of RAGE with a neutralizing antibody on the NS/PC surface, the ability of HMGB1 to enhance NS/PC proliferation was prevented. These findings suggest that RAGE also has an important function in the process of HMGB1-mediated NS/PC proliferation. Taguchi et al (30) reported that blocking RAGE-HMGB1mediated cellular stimulation decreased the levels of p-JNK, and further inhibited the proliferation of rat C6 glioma cells. In our experiments, blocking RAGE with an anti-RAGE antibody in HMGB1 cultures also decreased p-JNK. To further demonstrate that the JNK signaling pathway is involved in HMGB1-induced NS/PC proliferation, we used the potent JNK inhibitor, SP600125. We found that HMGB1 culture medium increased p-JNK levels and the rate of NS/PC proliferation. When the p-JNK levels in the NS/PCs were reduced by SP600125, the HMGB1 cultured NC/PC proliferation rates decreased correspondingly. All these results indicated that after binding to RAGE, HMGB1 increased NS/PC proliferation by promoting JNK phosphorylation.

Nevertheless, the present study also has certain limitations. Firstly, in these experiments, we stimulated astrocytes with low levels of IL-1 $\beta$ in vitro to mimic an in vivo inflammatory microenviroment, and confirmed that HMGB1 released from astrocytes promoted NS/PC proliferation in vitro. However, the actual inflammatory microenviroment is far more intricate, and whether this mechanism also exists in vivo requires further investigation. Secondly, astrocytes may also release other cytokines, such as nerve growth factor, neurotrophic factor, ciliary neurotrophic factor and vascular endothelial growth factor (39-42). The elucidation of the mechanisms through which HMGB1 interacts with these other networks and pathways requires further investigation. Thirdly, there is no doubt that the mechanism through which HMGB1 promotes NS/PC proliferation is very complex, and whether other signaling pathways, such as the ERK and p38 MAPK signaling pathways (43) are also involved in this process requires more detailed investigation in future studies.

In conclusion, our study provides evidence that HMGB1 released from reactive astrocytes is sufficient to promote NS/ PC proliferation, and the mechanisms through which HMGB1 promotes NS/PC proliferation may involve the activation of the RAGE-dependent JNK signaling pathway. Since the NS/PCs afford the plasticity to generate, repair and change nervous system function (44), the proliferation of NS/PCs may have beneficial effects in repairing brain damage. Our collective results support a previously described mechanism of a crosstalk between astrocytes and NS/PCs, and provide an experimental basis that reactive astrocyte-derived HMGB1 can promote NS/PC proliferation, which may be one of the reasons that NS/PCs spontaneously proliferate following brain injuries, and suggest that HMGB1 may be a very important factor for the regeneration of injured brain tissue. Further studies are required to investigate whether astrocyte-derived HMGB1 can induce NS/PCs to differentiate into neurons and to promote functional recovery following brain injury.

\section{Acknowledgements}

This study was supported by grants from the National Natural Science Foundation of China (no. 30470606) and the Program of the Traditional Chinese Medicine Research of Chongqing Municipal Health Bureau (no. 2003-B-16).

\section{References}

1. Go HS, Shin CY, Lee SH, et al: Increased proliferation and gliogenesis of cultured rat neural progenitor cells by lipopolysaccharide-stimulated astrocytes. Neuroimmunomodulation 16: 365-376, 2009.

2. Lee $\mathrm{C}, \mathrm{Hu}$ J, Ralls $\mathrm{S}$, et al: The molecular profiles of neural stem cell niche in the adult subventricular zone. PLoS One 7: e50501, 2012.

3. Cao X, Li LP, Qin XH, et al: Astrocytic adenosine 5'-triphosphate release regulates the proliferation of neural stem cells in the adult hippocampus. Stem Cells 31: 1633-1643, 2013.

4. Guo Y, Wei Q, Huang Y, Xia W, Zhou Y and Wang S: The effects of astrocytes on differentiation of neural stem cells are influenced by knock-down of the glutamate transporter, GLT-1. Neurochem Int 63: 498-506, 2013.

5. Zhao W, Wang Y, Shi W, et al: The expression of FBP1 after traumatic brain injury and its role in astrocyte proliferation. $J$ Mol Neurosci 51: 687-694, 2013.

6. Sukumari-Ramesh S, Alleyne CH Jr and Dhandapani KM: Astrocyte-specific expression of survivin after intracerebral hemorrhage in mice: a possible role in reactive gliosis? J Neurotrauma 29: 2798-2804, 2012

7. Hayakawa K, Pham LD, Katusic ZS, Arai K and Lo EH: Astrocytic high-mobility group box 1 promotes endothelial progenitor cell-mediated neurovascular remodeling during stroke recovery. Proc Natl Acad Sci USA 109: 7505-7510, 2012.

8. Kim JB, Lim CM, Yu YM and Lee JK: Induction and subcellular localization of high-mobility group box-1 (HMGB1) in the postischemic rat brain. J Neurosci Res 86: 1125-1131, 2008. 
9. Hayakawa K, Arai K and Lo EH: Role of ERK map kinase and CRM1 in IL-1 $\beta$-stimulated release of HMGB1 from cortical astrocytes. Glia 58: 1007-1015, 2010.

10. Sawa H, Ueda T, Takeyama Y, et al: Blockade of high mobility group box-1 protein attenuates experimental severe acute pancreatitis. World J Gastroenterol 12: 7666-7670, 2006.

11. Valdes-Ferrer SI, Rosas-Ballina M, Olofsson PS, et al: Highmobility group box 1 mediates persistent splenocyte priming in sepsis survivors: evidence from a murine model. Shock 40 : 492-495, 2013

12. Achouiti A, van der Meer AJ, Florquin S, et al: High-mobility group box 1 and the receptor for advanced glycation end products contribute to lung injury during Staphylococcus aureus pneumonia. Crit Care 17: R296, 2013.

13. Agnello D, Wang H, Yang H, Tracey KJ and Ghezzi P: HMGB-1, a DNA-binding protein with cy tokine activity, induces brain TNF and IL- 6 production, and mediates anorexia and taste aversion. Cytokine 18: 231-236, 2002.

14. Liu H, Yao YM, Ding LH, et al: High mobility group box-1 protein acts as a coactivator of nuclear factor of activated $\mathrm{T}$ cells-2 in promoting interleukin-2 transcription. Int J Biochem Cell Biol 41: 641-648, 2009.

15. Kim JB, Sig Choi J, Yu YM, et al: HMGB1, a novel cytokine-like mediator linking acute neuronal death and delayed neuroinflammation in the postischemic brain. J Neurosci 26: 6413-6421, 2006.

16. Lei $\mathrm{C}$, Lin $\mathrm{S}$, Zhang $\mathrm{C}$, et al: High-mobility group box1 protein promotes neuroinflammation after intracerebral hemorrhage in rats. Neuroscience 228: 190-199, 2013.

17. Laird MD, Shields JS, Sukumari-Ramesh S, et al: High mobility group box protein-1 promotes cerebral edema after traumatic brain injury via activation of toll-like receptor 4. Glia 62: 26-38, 2014.

18. Kim SW, Lim CM, Kim JB, et al: Extracellular HMGB1 released by NMDA treatment confers neuronal apoptosis via RAGE-p38 MAPK/ERK signaling pathway. Neurotox Res 20: 159-169, 2011.

19. Hayakawa K, Nakano T, Irie K, et al: Inhibition of reactive astrocytes with fluorocitrate retards neurovascular remodeling and recovery after focal cerebral ischemia in mice. J Cereb Blood Flow Metab 30: 871-882, 2010.

20. Hayakawa K, Miyamoto N, Seo JH, et al: High-mobility group box 1 from reactive astrocytes enhances the accumulation of endothelial progenitor cells in damaged white matter. J Neurochem 125: 273-280, 2013.

21. Guazzi S, Strangio A, Franzi AT and Bianchi ME: HMGB1, an architectural chromatin protein and extracellular signalling factor, has a spatially and temporally restricted expression pattern in mouse brain. Gene Expr Patterns 3: 29-33, 2003.

22. Zhao X, Kuja-Panula J, Rouhiainen A, Chen YC, Panula P and Rauvala H: High mobility group box-1 (HMGB1; amphoterin) is required for zebrafish brain development. J Biol Chem 286 23200-23213, 2011.

23. Meneghini V, Bortolotto V, Francese MT, et al: High-mobility group box- 1 protein and $\beta$-amyloid oligomers promote neuronal differentiation of adult hippocampal neural progenitors via receptor for advanced glycation end products/nuclear factor- $\kappa \mathrm{B}$ axis: relevance for Alzheimer's disease. J Neurosci 33: 6047-6059, 2013.

24. Nakagomi T, Taguchi A, Fujimori Y, et al: Isolation and characterization of neural stem/progenitor cells from post-stroke cerebral cortex in mice. Eur J Neurosci 29: 1842-1852, 2009.

25. Shen J, Xie L, Mao X, et al: Neurogenesis after primary intracerebral hemorrhage in adult human brain. J Cereb Blood Flow Metab 28: 1460-1468, 2008.

26. Itoh T, Imano M, Nishida $S$, et al: Exercise increases neural stem cell proliferation surrounding the area of damage following rat traumatic brain injury. J Neural Transm 118: 193-202, 2011.
27. Louis SA, Mak CK and Reynolds BA: Methods to culture, differentiate, and characterize neural stem cells from the adult and embryonic mouse central nervous system. Methods Mol Biol 946: 479-506, 2013.

28. Chen J, Guo Y, Cheng W, et al: High glucose induces apoptosis and suppresses proliferation of adult rat neural stem cells following in vitro ischemia. BMC Neurosci 14: 24, 2013.

29. Meneghini V, Francese MT, Carraro L and Grilli M: A novel role for the Receptor for Advanced Glycation End-products in neural progenitor cells derived from adult SubVentricular Zone. Mol Cell Neurosci 45: 139-150, 2010.

30. Taguchi A, Blood DC, del Toro G, et al: Blockade of RAGEamphoterin signalling suppresses tumour growth and metastases. Nature 405: 354-360, 2000.

31. Bao Y, Qin L, Kim E, et al: CD36 is involved in astrocyte activation and astroglial scar formation. J Cereb Blood Flow Metab 32: 1567-1577, 2012.

32. Jeong SR, Kwon MJ, Lee HG, et al: Hepatocyte growth factor reduces astrocytic scar formation and promotes axonal growth beyond glial scars after spinal cord injury. Exp Neurol 233: 312-322, 2012

33. Kawano H, Kimura-Kuroda J, Komuta Y, et al: Role of the lesion scar in the response to damage and repair of the central nervous system. Cell Tissue Res 349: 169-180, 2012.

34. Brambilla R, Morton PD, Ashbaugh JJ, Karmally S, Lambertsen KL and Bethea JR: Astrocytes play a key role in EAE pathophysiology by orchestrating in the CNS the inflammatory response of resident and peripheral immune cells and by suppressing remyelination. Glia 62: 452-467, 2014.

35. Palumbo R, Sampaolesi M, De Marchis F, et al: Extracellular HMGB1, a signal of tissue damage, induces mesoangioblast migration and proliferation. J Cell Biol 164: 441-449, 2004.

36. Limana F, Germani A, Zacheo A, et al: Exogenous high-mobility group box 1 protein induces myocardial regeneration after infarction via enhanced cardiac $\mathrm{C}-\mathrm{kit}^{+}$cell proliferation and differentiation. Circ Res 97: e73-e83, 2005.

37. Wang X, Fu S, Wang Y, et al: Interleukin-1 $\beta$ mediates proliferation and differentiation of multipotent neural precursor cells through the activation of SAPK/JNK pathway. Mol Cell Neurosci 36: 343-354, 2007

38. Kim J, Wan CK, S JOC, Shaikh SB and Nicholson LF: The role of receptor for advanced glycation end products (RAGE) in neuronal differentiation. J Neurosci Res 90: 1136-1147, 2012.

39. Yoshida H, Mimura J, Imaizumi T, et al: Edaravone and carnosic acid synergistically enhance the expression of nerve growth factor in human astrocytes under hypoxia/reoxygenation. Neurosci Res 69: 291-298, 2011.

40. Koyama Y, Maebara Y, Hayashi M, Nagae R, Tokuyama S and Michinaga S: Endothelins reciprocally regulate VEGF-A and angiopoietin-1 production in cultured rat astrocytes: implications on astrocytic proliferation. Glia 60: 1954-1963, 2012.

41. Kuric E, Wieloch T and Ruscher K: Dopamine receptor activation increases glial cell line-derived neurotrophic factor in experimental stroke. Exp Neurol 247: 202-208, 2013.

42. Modi KK, Sendtner M and Pahan K: Up-regulation of ciliary neurotrophic factor in astrocytes by aspirin: implications for remyelination in multiple sclerosis. J Biol Chem 288: 18533-18545, 2013.

43. Tian Y, Liu Y, Chen X, et al: AMN082 promotes the proliferation and differentiation of neural progenitor cells with influence on phosphorylation of MAPK signaling pathways. Neurochem Int 57: 8-15, 2010.

44. Gage FH and Temple S: Neural stem cells: generating and regenerating the brain. Neuron 80: 588-601, 2013. 